\title{
Histone Deacetylase Inhibitors: A Novel Therapeutic Weapon Against Medullary Thyroid Cancer?
}

\author{
CHRISTOS DAMASKOS ${ }^{1,2 *}$, SERENA VALSAMI ${ }^{3 *}$, ELEFTHERIOS SPARTALIS $^{2}$, \\ EFSTATHIOS A. ANTONIOU ${ }^{1}$, PERIKLIS TOMOS ${ }^{4}$, STEFANOS KARAMAROUDIS $^{5}$, \\ THEOFANO ZOUMPOU ${ }^{5}$, VASILIOS PERGIALIOTIS ${ }^{2}$, KONSTANTINOS STERGIOS ${ }^{2,6}$, \\ CONSTANTINOS MICHAELIDES ${ }^{7}$, KONSTANTINOS KONTZOGLOU ${ }^{1}$, DESPINA PERREA ${ }^{2}$, \\ NIKOLAOS NIKITEAS ${ }^{2}$ and DIMITRIOS DIMITROULIS ${ }^{1}$ \\ ${ }^{1}$ Second Department of Propedeutic Surgery, "Laiko” General Hospital, Medical School, \\ National and Kapodistrian University of Athens, Athens, Greece; \\ ${ }^{2}$ Laboratory of Experimental Surgery and Surgical Research N.S. Christeas, Medical School, \\ National and Kapodistrian University of Athens, Athens, Greece; \\ ${ }^{3}$ Blood Transfusion Department, Aretaieion Hospital, Medical School, \\ National and Kapodistrian Athens University, Athens, Greece; \\ ${ }^{4}$ Department of Thoracic Surgery, “Attikon” General Hospital, Medical School, \\ National and Kapodistrian University of Athens, Athens, Greece; \\ ${ }^{5}$ Medical School, National and Kapodistrian University of Athens, Athens, Greece; \\ ${ }^{6}$ Colorectal Department, General Surgery, The Princess Alexandra Hospital NHS Trust, Harlow, U.K.; \\ ${ }^{7} 1$ st Department of Pathology, School of Medicine, University of Athens, Athens, Greece
}

\begin{abstract}
Background/Aim: Medullary thyroid cancer (MTC) is highly malignant, metastatic and recurrent, remaining generally incurable, and responsible for approximately $14 \%$ of all thyroid carcinoma-related deaths. MTC can metastasize to lymph nodes, trachea and distant organs, such as brain, lungs, liver and bones. MTC cells are resistant to chemotherapy and traditional external therapies are not showing definite clinical benefits. Scientists are trying to understand the molecular background of carcinogenesis
\end{abstract}

This article is freely accessible online.

\footnotetext{
*These Authors contributed equally to this study.

Correspondence to: Christos Damaskos, MD, MSc, Ph.D., Second Department of Propedeutic Surgery, "Laiko" General Hospital, National and Kapodistrian University of Athens, Medical School, Athens, Greece; Laboratory of Experimental Surgery and Surgical Research N.S. Christeas, National and Kapodistrian University of Athens, Medical School, Athens, Greece, 17 Agiou Thoma Street, Athens, 11527, Greece. Tel: +30 6948467790, Fax: +30 2107456972, e-mail: x_damaskos@yahoo.gr
}

Key Words: HDAC, histone, inhibitors, acetylasation, epigenetics, medullary, thyroid, cancer, targeted, therapy, review. and histone deacetylase (HDAC) seems to play a potential role to gene transcription. On the other hand, HDAC inhibitors (HDACI) hamper the HDAC action giving promising results as new anticancer drugs. The purpose of this review was to evaluate the current status of research considering the role of HDACIs in MTC treatment and to present the latest trends in MTC treatment protocols. Materials and Methods: This literature review was accomplished using the MEDLINE database. The key words/phrases were; HDACI, medullary thyroid cancer, HDACI in the therapy of neuroendocrine tumors, HDACI in MTC. Fortyone articles were selected from the total number of the search's results. Only sixteen papers focus on the use of HDACIs in the treatment of MTC. In order to extract our conclusions, we took into account some studies whose main topic does not strictly refer to the MTC but they contain noteworthy and useful information. Only English articles published up to August 2016 were assessed and used for writing this review. Results: Molecules, such as valproid acid (VPA), vorinostat, suberoyl bis-hydroxamic acid (SBHA), depsipeptide, belinostat, m-carboxycinnamic acid bishydroxamine (CBHA) and $A B 3$ have shown promising antitumor effects against MTC. Conclusion: HDACIs represent a promising field for targeted therapy both for its anticancer properties, as well as for augmenting radiotherapeutic modalities. More trials are needed. 
Medullary thyroid cancer (MTC) is a malignant tumor originating from the parafollicular C-cells of the neural crest (1). It belongs to the neuroendocrine (NE) neoplasms and, more specifically, secretes primarily calcitonin (2) but also chromogranin A (CgA), synaptophysin (SYP), achaete-scute complex-like 1 (ASCL1), carcinoembryonic antigen, neuronspecific enolase, serotonin, gastrin-releasing peptide, substance $\mathrm{P}$, pro-opiomelacortin-derived products and somatostatin. Patients present with hormone-related symptoms, such as diarrhea, flushing, ectopic corticotrophin syndrome, electrolytic disorders and Cushing syndrome (3).

MTC represents $3 \%$ of all thyroid malignancies and is highly malignant, metastatic and recurrent remaining, generally, incurable (4-6). However, it is responsible for approximately $14 \%$ of all thyroid carcinoma-related deaths (5). Roughly half of all MTCs metastasize; lymph node metastases are frequent, but MTCs can also easily invade the jugular vein or the trachea and give metastases to more distant organs, such as the brain, the lungs, the liver and the bones (7-9). Additional symptoms of advanced and metastatic MTCs are airway obstruction and abdominal pain (3).

MTC consists of slowly-growing cells, thus being resistant to chemotherapy that, typically, targets fast replicating cells. In addition, due to their neuroendocrine nature, MTC cells do not respond to radioiodine or thyroid-stimulating hormone (TSH)-suppressive therapy. Therefore, mostly palliative therapies can be used to alleviate patients' symptoms, including radiotherapy, chemotherapy, symptomtargeted therapy, radiopeptide therapy, radioiodinated metaiodobenzylguanidine (MIBG) treatment, etc. $(5,6,9,10)$.

A rate of $70 \%$ of MTC appears in a sporadic manner, while the rest $30 \%$ of the cases belong to familial forms. There are 3 main familial forms; MEN2A, MEN2B and familial MTC not associated with MEN (5). MEN2A is characterized by MTC, pheochromocytoma and parathyroid hyperplasia, whereas MEN2B includes MTC, pheochromocytoma, ganglioneuroma, mucosal neuroma and marfanoid habitus (11).

The emergence of MTC is associated with genetic mutations. One such identified mutation is a chromosomal rearrangement linking the promoter and $\mathrm{N}$-terminal of an unrelated gene(s) to the C-terminal of the RET protooncogene (receptor with tyrosine kinase activity) $(11,12)$. Scientists are trying to reveal the molecular profile of MTCs and transfer the battle with MTC to the molecular level.

A promising treatment strategy is inhibiting histone deacetylases (HDACs) and many novel molecules with HDAC inhibitory activity are currently being tested. DNA is wrapped around proteins called histones. HDACs are a class of enzymes that remove acetyl groups from histone lysine residues. Histone deacetylation allows DNA to wrap more tightly, thus becoming less accessible to the cell's transcription machine $(1,7)$. More specifically, HDACs suppress the expression of tumor-suppressor genes and promote, in this way, the development and progression of tumors (13). In addition, HDAC activity is enhanced in both malignant and benign neoplasms (7). There are several HDAC groups among which HDAC-1, -4, -6 are related to tumor size; HDAC is also related to capsular invasion and HDAC-2 is related to lymphatic and vascular invasion in malignant neoplasms (14). It is now obvious why the use of HDAC inhibitors (HDACIs) appears promising in treating various neoplasms.

HDACIs can promote tumor growth arrest, differentiation and apoptosis (15). What is more, they can sensitize tumor cells to radiation and chemotherapy (7) and make tumors more amenable to surgery (13). More specifically, HDACIs have been shown to synergize with antitumor/chemotherapy drugs, such as paclitaxel, cisplatin, etoposide, doxorubicin, the HAMLET protein complex, etc. Moreover, HDACIs modulate the expression of microRNAs (miRNAs). miR-129-5p, for example, is necessary in order for HDACI-induced and drugcell death to be achieved. Of course, the most obvious effect of HDACIs is that the NH2 terminal of histones' lysine residues remains acetylated, which gives DNA a more open configuration. This is important as it allows key genes (e.g., tumor-suppressor genes) to be expressed (13).

Recently, HDACI entered clinical trials, particularly for patients who have already been treated and have multiple relapses in advanced disease (16-21). The first HDACIs submitted to tests and clinical studies have shown promising antitumor effects at doses tolerated by patients.

Zolinza $^{\circledR}$ (vorinostat) constitutes the first HDACI that received approval for clinical use for the treatment of patients with hematologic neoplasia (cutaneous T-cell lymphoma) (22). Currently made therapy studies with HDACIs, either alone or in combination with cytotoxic or targeted cancer treatments, as well as at least ten different treatments with HDACIs, are in phase II or III clinical trials for the treatment of tumors, hematologic or other (16-21). Unfortunately, until now published data for the immunohistological expression of HDAC in MTC are poor. Research focuses on the following molecules; valproic acid, butyrates, trichostatin A, depsipeptide, vorinostat (SAHA), suberoyl bis-hydroxamic acid (SBHA), belinostat and $\mathrm{AB} 3$, to which we will make a detailed reference. Major actions of these agents are summarized in Table I.

\section{Valproic Acid}

Valproic (VPA) acid is a branched fatty acid. It has anticonvulsant activity and is currently being used for treating bipolar disorders, epilepsy, seizures, depression and other neuropsychiatric disorders (8). VPA is also a selective class I HDACI and was proposed for treating malignancies, including thyroid neoplasms, neuroblastoma and hematological malignancies. The use of VPA in vitro (doses of $0.5-3 \mathrm{mmol} / \mathrm{l}$ ) causes growth inhibition, cell-cycle arrest at G1 phase and 
Table I. Histone deacetylase inhibitors (HDACIs) as antineoplasmatic agents and their action in medullary thyroid cancer (MTC).

\begin{tabular}{lc}
\hline Substance & Action \\
\hline Valproic Acid & Growth inhibition, cell cycle arrest at G1 phase and apoptosis \\
Butyrates & Inhibition of cell proliferation in a dose-dependent manner \\
Trichostatin A & Inhibition and tumor suppression \\
Depsipeptide & Reversion of the tumor cells' radioiodine resistance \\
Vorinostat (SAHA) & Tumor growth arrest and apoptosis \\
Suberoyl bis-hydroxamic acid (SBHA) & Antiproliferative/apoptotic activity \\
Belinostat & Inhibition of cell proliferation in a dose-dependent manner \\
AB3 & Inhibition of cell proliferation/apoptosis \\
\hline
\end{tabular}

apoptosis. Apoptosis was induced by down-regulation of prosurvival/antiapoptotic genes $\mathrm{BCL}-2$ and $\mathrm{BCL}-\mathrm{X}_{\mathrm{L}}$ and upregulation of the pro-apoptotic $B A X$ gene. Furthermore, VPA enhanced sodium/iodide symporter (NIS) expression and, subsequently, increased the radioiodine uptake for tumor cells in vitro.

Regarding thyroid malignancies, in the study of Yuan et al., the effect of VPA on the inhibition of RET signaling and induction of apoptosis in human thyroid carcinoma cells (cell lines) was investigated. VPA induced metabolic stress, activated AMP-activated protein kinase and increased autophagic flux. Expression of VPA molecular targets in metastatic human thyroid carcinoma cells suggests that VPA has a potential to become a thyroid cancer therapeutic agent (23).

Moreover, in vitro tumor cell treatment with VPA was related to the Notch1 pathway activation. Notch1 promotes tumor suppression, growth inhibition in vivo and in vitro and overall decrease in NE markers (e.g., calcitonin, $\mathrm{CgA}$, ASCL1) $(5,8)$. In a phase I clinical trial, papillary thyroid carcinoma (PTC) metastatic patients treated with VPA and 5-azacytidine remained stable for approximately 12 months, whereas MTC patients did not respond to treatment.

Another therapeutic combination of VPA (or SBHA) and lithium chloride was shown to be more effective on lower doses (5).

\section{Vorinostat}

Vorinostat or suberoyl-anilide hydroxamic acid (SAHA) is composed from novel synthetic hybrid polar compounds. It is an active class I and II HDACI that can be received orally. It has an excellent bioavailability and has been approved by the US FDA for treating cutaneous T-cell lymphoma. It targets class I and II HDACs and binds directly to their active site when $\mathrm{Zn}^{+2}$ is present (7).

In preclinical studies, vorinostat induced tumor growth arrest and apoptosis by cleaving the Poly(ADP-ribose) polymerase (PARP), i.e. caspaces $-2,-3,-7,-8,-9$. It also induced cell-cycle arrest at $\mathrm{G}_{1}$ phase, early up-regulation of cyclin-dependent kinase (CDK) inhibitor p21, decrease of phosphorylation of $\mathrm{CDK}$ substrate retinoblastoma and increased tumor cells' sensitivity $(1,7)$. Furthermore, there are signs of synergistic relationship with doxorubicin, paclitaxel and paraplatin. Finally, VPA, when administered with trichostatin A, induced the overexpression of miR-129-5p (13) (which increases the antiproliferative effect of various cancer drugs), histone acetylation and cell death. The maximum dose that can be administered to patients is $200 \mathrm{mg}$ b.i.d. and, till this point, there are no clinical responses. A phase II study failed to show any useful results and was terminated for some patients due to adverse effects (7).

\section{Depsipeptide}

Depsipeptides (e.g., FR901228, NSC630176) are bicyclic peptides produced by fermentation from Chromobacterium violaceum (1). Ninety percent of depsipeptide circulates in a protein-bound form. It has a strong cytotoxic activity against various human cancer cell lines, such as cutaneous or peripheral T-cell lymphoma, renal cell carcinoma, etc. As far as thyroid cells are concerned, depsipeptide up-regulates the expression of thyroglobulin and NIS. The increase in the number of NIS increases the cell's capacity for I ${ }^{125}$ uptake and, thus, can reverse the tumor cells' radioiodine resistance (24). There are no responses from clinical trials so far, whereas one death, one grade 4 pulmonary embolus and 12 more patients with adverse effects have been recorded. Depsipeptide causes no significant toxicity at a dose of $500 \mathrm{ng} / \mathrm{ml}$. Fatigue, nausea, vomiting, neutropenia and transient thrombocytopenia are all side-effects of depsipeptide treatment (25).

\section{Belinostat}

Belinostat (PXD101) inhibits cell proliferation in a dosedependent manner. More specifically, a dose of $1.25 \mu \mathrm{mol} / 1$ repressed tumor growth up to $45 \%$ in MTC, while a $10 \mu \mathrm{mol} / \mathrm{l}$ dose caused a 95\% tumor repression. PXD101 inhibits class I, IIa and IIb HDACs and causes acetylation of H3, H4 and 
tubulin. It also leads to apoptosis by accumulation of reactive oxygen species (ROS). Additionally, belinostat promotes DNA damage and, more specifically, double-stranded DNA breaks (DSBs). p-H2AX (Ser139), a DSB marker, is increased. At the same time, non-homologous end joining (NHEJ) DNA repair proteins KU70, KU80 and RAD51 (the pivotal homologous recombination repair protein) are down-regulated (26).

\section{m-Carboxycinnamic Acid bis-Hydroxamine}

The m-carboxycinnamic acid bis-hydroxamine (CBHA) has, in general, the same results with SAHA. It promotes growth arrest and caspace-mediated apoptosis. Retinoblastoma $(R B)$ tumor suppressor gene is hypophosphorylated, p21 and BAX protein are up-regulated, while antiapoptotic proteins BCL-2, BCLA1 and $\mathrm{BCL}-\mathrm{X}_{\mathrm{L}}$ are down-regulated. CBHA activity is also related to the cleavage of PARP and BH3-interacting death agonist (1).

\begin{abstract}
AB3
AB3 is a novel class I HDACI. It induces the Notch3 pathway and activates the Notch3 mediator C-promoter binding factor 1 (CBF1). In this way, apoptotic events are induced, thus inhibiting cell proliferation in MTC. Notably, the transcription of the $H E S / H E Y$ gene family is altered; HES1, HES5, HEY1, HEY2 are up-regulated and HES2 and HES6 are down-regulated. AB3 is also responsible for PARP cleavage, XIAP, surviving and p21 suppression, as well as p27 enhancement, ending up with cell-cycle arrest and sub$\mathrm{G}_{1}$ DNA accumulation. AB3 shows the highest inhibitory activity at doses of 1-10 $\mu \mathrm{mol} / 1$ (best: $2 \mu \mathrm{mol} / \mathrm{l}$ ) (3).
\end{abstract}

\section{Butyrate}

Butyrate is a short-chain fatty acid produced by anaerobic bacterial fermentation of dietary fibers. Butyrate alters gene expression and blocks cell proliferation by inhibition of the chromatin-remodeling activity of HDAC, while it increases iodine uptake $(27,28)$. It is suggested that butyrate regulates gene expression using ATP citrate lyase (ACL)-dependent and -independent mechanisms. Butyrate is an attractive agent for chemotherapy or chemoprevention because it selectively inhibits tumor growth over normal cells without gross adverse effects (28). Regarding thyroid malignancies, it has been found that butyrate and TSA repressed RAP activity in anaplastic carcinoma cell lines (30). Nevertheless, butyrate's rapid uptake and metabolism limit its efficacy (29).

\section{Tricostatin A}

Trichostatin A (TSA) is an antifungal drug that can inhibit HDACs leading functional genes to active or to inhibited states and regulating their transcription and expression. TSA might act on the pre-regulation of DNA in transcription, inhibit HDACs and open the DNA chain through the acetylation of histones. TSA might act alone or in combination with other agents $(31,32)$. It is suggested that TSA restores the expression of Rap1GAP in thyroid tumor cells resulting in Rap activity inhibition and tumor suppression (33).

\section{Suberoyl bis-Hydroxamic Acid}

Suberoyl bis-hydroxamic acid (SBHA), like VPA, increases the amount of active Notch1 protein (5). In addition, it promotes the up-regulation of $B A X, B A D$ and $B M F$ genes and increases the amount of $\mathrm{p} 21^{\mathrm{WAF} 1 / \mathrm{CIP} 1}, \mathrm{p} 27^{\mathrm{KIP} 1}$, cleaved caspaces- 3 and -9 and cleaved PARP, all of which have an antiproliferative/apoptotic activity. Moreover, SBHA treatment results in a decrease of the NE markers $\mathrm{CgA}$ and ASCL1, as well as decrease of cyclins B1 and D1 (mitotic cyclins), $B C L-2$ (oncogene) and $B C L-\mathrm{X}_{\mathrm{L}}$ (apoptotic inhibitor). In an experimental study, where mice were treated with $200 \mathrm{mg} / \mathrm{kg}$ for 12 days, tumor growth was inhibited up to $55 \%$ (34).

\section{Conclusion}

Changes in gene expression or cellular phenotype caused by mechanisms other than changes in DNA sequence are called epigenetic changes and they are identified as an important cause of chemoresistence. DNA methylation and chromatin remodeling, RNA transcripts and their encoded proteins, chromatin and mRNA regulation are vulnerable to change and histone acetylation and deacetylation is a major mechanism modifying gene expression. Indeed, alterations in histone acetylation exert crucial changes on chromatin condensation and gene transcription. An imbalance between histone transacetylases and deacetylases is often present in cancer, damaging expressions of tumor suppressor genes and/or proto-oncogenes $(33,35,36)$. The acetylation state of histones and other proteins is maintained by histone acetyltransferase (HAT) and histone deacetylase (HDAC) enzymes. The inhibition of these enzymes affect various cell functions. However, HDAC inhibitors are extremely specific for cancer cells over normal cells, as a result of proapoptotic genes' induction besides their effects on DNA repair mechanisms. Their action against cancer cells, involve apoptosis, autophagy, growth inhibition and differentiation (35).

Several HDAC inhibitors are under clinical development and two of them, vorinostat and romidespin, have been approved by the US FDA for treating patients with cutaneous T-cell lymphoma (CTCL) $(35,36)$. HDAC inhibitors also appear to be active in acute myeloid leukemia (AML), lymphomas and myelodysplastic syndromes (MDS). 
However, in studies involving solid tumors, their efficacy is questionable. For thyroid cancer, VPA is a potent candidate, as in other solid malignancies as well $(23,37)$.

The association of MTC with mutations in the RET protooncogene and the overexpression of HDACs in thyroid carcinomas allow a field of action for HDACs, which have emerged as potent, very promising therapeutic agents for the thyroid cancer. They could be utilized either individually or in combination with other anticancer drugs $(23,30,38)$. They could be especially valuable in MTC, due to disappointing prognosis as RET-positive MTC patients are found to have increased probability of persistent disease and a lower survival rate (39). Of note, thyroid carcinomas (MTC) comprise only 3-5\% of thyroid malignancies but they are responsible for $14 \%$ of all thyroid cancer deaths. In this context, the potential value of HDACs is evident $(40,41)$. This fact justifies extensive research in the field because, despite their abundance, explanations of their actions remain grossly unclear. Also, their use in combination with other drugs need detailed investigation and the question whether pan-HDAC inhibitors or selective inhibitors are more efficient in different types of cancers is yet to be answered. Especially for solid tumors, there is an urgent need of novel HDAC therapeutic approaches.

\section{References}

1 Mitsiades CS, Poulaki V, McMullan C, Negri J, Fanourakis G, Goudopoulou A, Richon VM, Marks PA and Mitsiades N: Novel histone deacetylase inhibitors in the treatment of thyroid cancer. Clin Cancer Res 11: 3958-3965, 2005.

2 Raue F and Frank-Raue K: Epidemiology and Clinical Presentation of Medullary Thyroid Carcinoma. Recent Results Cancer Res 204: 61-90, 2015.

3 Jaskula-Sztul R, Eide J, Tesfazghi S, Dammalapati A, Harrison AD, Yu XM, Scheinebeck C, Winston-McPherson G, Kupcho KR, Robers MB, Hundal AK, Tang W and Chen H: Tumorsuppressor role of Notch3 in medullary thyroid carcinoma revealed by genetic and pharmacological induction. Mol Cancer Ther 14: 499-512, 2015.

4 Sugawara M, Geffner DL, Martinez D and Hershman JM: Novel treatment of medullary thyroid cancer. Curr Opin Endocrinol Diabetes Obes 16: 367-372, 2009.

5 Adler JT, Hottinger DG, Kunnimalaiyaan $M$ and Chen H: Inhibition of growth in medullary thyroid cancer cells with histone deacetylase inhibitors and lithium chloride. J Surg Res 159: 640-644, 2010.

6 Biermann K, Biersack HJ, Sabet A and Janzen V: Alternative therapeutic approaches in the treatment of primary and secondary dedifferentiated and medullary thyroid carcinoma. Semin Nucl Med 41: 139-148, 2011.

7 Woyach JA, Kloos RT, Ringel MD, Arbogast D, Collamore M, Zwiebel JA, Grever M, Villalona-Calero M and Shah MH: Lack of therapeutic effect of the histone deacetylase inhibitor vorinostat in patients with metastatic radioiodine-refractory thyroid carcinoma. J Clin Endocrinol Metab 94: 164-170, 2009.
8 Greenblatt DY, Cayo MA, Adler JT, Ning L, Haymart MR, Kunnimalaiyaan $\mathrm{M}$ and Chen $\mathrm{H}$ : Valproic acid activates Notch1 signaling and induces apoptosis in medullary thyroid cancer cells. Ann Surg 247: 1036-1040, 2008.

9 Smit J: Treatment of advanced medullary thyroid cancer. Thyroid Res 6(Suppl 1): S7, 2013.

10 Sung TY, Choi SH, Lee JM, Jeong JJ, Kang SW and Chung WY: Innovative in vitro chemo-hormonal drug therapy for refractory thyroid carcinomas. J Korean Med Sci 27: 729-735, 2012.

11 Giunti S, Antonelli A, Amorosi A and Santarpia L: Cellular signaling pathway alterations and potential targeted therapies for medullary thyroid carcinoma. Int J Endocrinol 2013: 803171, 2013.

12 Milano A, Chiofalo MG, Basile M, Salzano de Luna A, Pezzullo L and Caponigro F: New molecular targeted therapies in thyroid cancer. Anticancer Drugs 17: 869-879, 2006.

13 Brest P, Lassalle S, Hofman V, Bordone O, Gavric Tanga V, Bonnetaud C, Moreilhon C, Rios G, Santini J, Barbry P, Svanborg C, Mograbi B, Mari B and Hofman P: MiR-129-5p is required for histone deacetylase inhibitor-induced cell death in thyroid cancer cells. Endocr Relat Cancer 18: 711-719, 2011.

14 Giaginis C, Alexandrou P, Delladetsima I, Giannopoulou I, Patsouris $\mathrm{E}$ and Theocharis S: Clinical significance of histone deacetylase (HDAC)-1, HDAC-2, HDAC-4, and HDAC-6 expression in human malignant and benign thyroid lesions. Tumour Biol 35: 61-71, 2014.

15 Damaskos C, Karatzas T, Nikolidakis L, Kostakis ID, Karamaroudis S, Boutsikos G, Damaskou Z, Kostakis A and Kouraklis G: Histone deacetylase (HDAC) inhibitors: Current evidence for therapeutic activities in pancreatic cancer. Anticancer Res 35: 3129-3136, 2015.

16 Khan $\mathrm{O}$ and La Thangue NB: HDAC inhibitors in cancer biology: Emerging mechanisms and clinical applications. Immunol Cell Biol 90: 85-94, 2012.

$17 \mathrm{Ma} \mathrm{X}$, Ezzeldin HH and Diasio RB: Histone deacetylase inhibitors: current status and overview of recent clinical trials. Drugs 69: 1911-1934, 2009.

18 Tan J, Cang S, Ma Y, Petrillo RL and Liu D: Novel histone deacetylase inhibitors in clinical trials as anti-cancer agents. J Hematol Oncol 3: 5, 2010.

19 Giaginis C, Damaskos C, Koutsounas I, Zizi-Serbetzoglou A, Tsoukalas N, Patsouris E, Kouraklis G and Theocharis S: Histone deacetylase (HDAC)-1, -2, -4 and -6 expression in human pancreatic adenocarcinoma: Associations with clinicopathological parameters, tumor proliferative capacity and patients' survival. BMC Gastroenterol 15: 148, 2015.

20 Lane AA and Chabner BA: Histone deacetylase inhibitors in cancer therapy. J Clin Oncol 27: 5459-5468, 2009.

21 Molife LR and de Bono JS: Belinostat: Clinical applications in solid tumors and lymphoma. Expert Opin Investig Drugs 20: 1723-1732, 2011.

22 National Institutes of Health (NIH), Clinical Trials. Available at: http://www.clinicaltrials.gov.

$23 \mathrm{Xu} \mathrm{Y,} \mathrm{Xu} \mathrm{D,} \mathrm{Zhu} \mathrm{SJ,} \mathrm{Ye} \mathrm{B,} \mathrm{Dong} \mathrm{JD,} \mathrm{Zhang} \mathrm{YL} \mathrm{and} \mathrm{Zhang} \mathrm{Y:}$ Induction of apoptosis and autophagy in metastatic thyroid cancer cells by valproic acid (VPA). Int J Clin Exp Pathol 8: 8291-8297, 2015.

24 Provenzano MJ, Fitzgerald MP, Krager K and Domann FE: Increased iodine uptake in thyroid carcinoma after treatment with sodium butyrate and decitabine (5-Aza-dC). Otolaryngol Head Neck Surg 137: 722-728, 2007. 
25 Whitehead RP, Rankin C, Hoff PM, Gold PJ, Billingsley KG, Chapman RA, Wong L, Ward JH, Abbruzzese JL and Blanke CD: Phase II trial of romidepsin (NSC-630176) in previously treated colorectal cancer patients with advanced disease: A Southwest Oncology Group study (S0336). Invest New Drugs 27: 469-475, 2009.

26 Lin SF, Lin JD, Chou TC, Huang YY and Wong RJ: Utility of a histone deacetylase inhibitor (PXD101) for thyroid cancer treatment. PLoS One 8: e77684, 2013.

27 Provenzano MJ, Fitzgerald MP and Domann FE: Increased iodine uptake in thyroid carcinoma after treatment with sodium butyrate and decitabine (5-Aza-dC). Otolaryngol Head Neck Surg 137: 722-728, 2007.

28 Davie JR: Inhibition of histone deacetylase activity by butyrate. J Nutr 133(7 Suppl): 2485S-2493S, 2003.

29 Pouillart PR: Role of butyric acid and its derivatives in the treatment of colorectal cancer and hemoglobinopathies. Life sciences 63: 1739-1760, 1998.

30 Dong X, Korch C and Meinkoth JL: Histone deacetylase inhibitors upregulate Rap1GAP and inhibit Rap activity in thyroid tumor cells. Endocr Relat Cancer 18: 301-10, 2011.

31 Yuan GB, Kuang AR, Fan Q, Yu LB and Mi YX; Combined effects of all-trans-retinoic acid and trichostatin $\mathrm{A}$ on the induction of differentiation of thyroid carcinoma cells. Chin J Cancer 29: 379-84, 2010.

32 Zarnegar R, Brunaud L, Kanauchi H, Wong M, Fung M, Ginzinger D, Duh QY and Clark OH: Increasing the effectiveness of radioactive iodine therapy in the treatment of thyroid cancer using Trichostatin A, a histone deacetylase inhibitor. Surgery 132: 984-90, 2002.

33 Hrabeta J, Stiborova M, Adam V, Kizek R and Eckschlager T: Histone deacetylase inhibitors in cancer therapy. A review. Biomed Pap Med Fac Univ Palacky Olomouc Czech Repub 158: 161-169, 2014.
34 Ning L, Jaskula-Sztul R, Kunnimalaiyaan $\mathrm{M}$ and Chen $\mathrm{H}$ : Suberoyl bishydroxamic acid activates notch1 signaling and suppresses tumor progression in an animal model of medullary thyroid carcinoma. Ann Surg Oncol 15: 2600-2605, 2008.

35 Zwergel C, Stazi G, Valente S and Mai A: Histone deacetylase inhibitors: Updated studies in various epigenetic-related diseases. J Clin Epigenet 2: 1, 2016.

$36 \mathrm{Kim}$ HJ and Bae SC: Histone deacetylase inhibitors: Molecular mechanisms of action and clinical trials as anti-cancer drugs. Am J Transl Res 3: 166-179, 2011.

37 Scherpereel A, Berghmans T, Lafitte JJ, Colinet B, Richez M, Bonduelle Y, Meert AP, Dhalluin X, Leclercq N, Paesmans M, Willems L and Sculier JP: Valproate-doxorubicin: Promising therapy for progressing mesothelioma. A phase II study. Eur Respir J 37: 129-135, 2011.

38 Borbone E, Berlingieri MT, De Bellis F, Nebbioso A, Chiappetta G, Mai A, Altucci L and Fusco A: Histone deacetylase inhibitors induce thyroid cancer specific apoptosis through proteasomedependent inhibition of TRAIL degradation. Oncogene 29: 105$116,2010$.

39 Wells SA Jr. and Santoro M: Targeting the RET pathway in thyroid cancer. Clin Cancer Res 15: 7119-7123, 2009.

40 Hyer SL, Newbold K and Harmer C: Familial medullary thyroid cancer clinical aspects and prognosis. Eur J Surg Oncol 31: 415419, 2005.

41 Cook M, Yu XM and Chen H: Notch in the development of thyroid C-cells and the treatment of medullary thyroid cancer. Am J Transl Res 2: 119-125, 2010.

Received September 1, 2016

Revised September 15, 2016

Accepted September 19, 2016 